\title{
Dual-Modality All-Optical Ultrasound and Photoacoustic Imaging Using Permanent Marker Ink
}

\author{
Richard J Colchester ${ }^{*,}$, Sacha Noimark ${ }^{*, \dagger}$, , Edward Z Zhang*, Paul C Beard*, ${ }^{*}$, and Adrien E Desjardins ${ }^{*, \dagger}$ \\ *Department of Medical Physics \& Biomedical Engineering, University College London, London, United Kingdom \\ ${ }^{\dagger}$ Wellcome / EPSRC Centre for Surgical and Interventional Sciences, University College London, London, United Kingdom \\ ${ }_{\ddagger}^{\ddagger}$ Materials Chemistry Research Centre, Department of Chemistry, University College London, London, United Kingdom \\ E-mail: richard.colchester@ucl.ac.uk
}

\begin{abstract}
A dual-modality all-optical ultrasound and photoacoustic imaging probe was fabricated using a commercially available permanent marker ink. The permanent marker ink was dried and mixed with polydimethylsiloxane to form a composite coating on optical fiber tips. The coating exhibited wavelengthselective absorption, with absorption $>90 \%$ at $532 \mathrm{~nm}$ for ultrasound generation, and $<10 \%$ at $1064 \mathrm{~nm}$ for photoacoustic light transmission. Ultrasound generated by the device had a peak-to-peak pressure in excess of $200 \mathrm{kPa}$ and a corresponding $-6 \mathrm{~dB}$ bandwidth of $17.3 \mathrm{MHz}$. The fabricated transmitter was combined with a plano-concave Fabry-Pérot fiber optic hydrophone to create an imaging probe. The axial ultrasound resolution of the probe was $<200 \mu \mathrm{m}$. Co-registered ultrasound and photoacoustic imaging was demonstrated with a wire phantom. This work demonstrates the feasibility for making low-cost dual-modality devices using all-optical ultrasound.

Index Terms-All-optical ultrasound, photoacoustic imaging, ultrasound imaging, polydimethylsiloxane, composite
\end{abstract}

\section{INTRODUCTION}

Recently there has been interest in the use of miniature hybrid probes for guiding minimally invasive procedures [1] [3]. One promising hybrid is the combination of ultrasound and photoacoustic imaging [4]-[9]. With ultrasound imaging, an ultrasound wave is transmitted into the tissue and the received reflections are used to generate an image, thereby providing structural contrast. With photoacoustic imaging, light is delivered to the tissue where it is selectively absorbed and generates an ultrasound wave which is received, providing molecular contrast. These two complementary modalities have been realised using electronic ultrasound transducers [4] [9]. However, alternatives to electronic ultrasound transducers are desirable to achieve increased bandwidth, compactness, flexibility and immunity to electromagnetic interference.

All-optical ultrasound, where ultrasound is generated and received using light, is emerging as a viable solution. Ultrasound generation is performed via the photoacoustic effect, wherein pulsed or modulated laser light is absorbed within a coating

This work was supported by an ERC Starting Grant [310970 MOPHIM], an ERC Advanced Grant [741149], the Innovative Engineering for Healthcare award by Wellcome Trust [WT101957] and the EPSRC [NS/000027/1], the EPSRC Healthcare Technologis Challenge Award [EP/N021177/1], and the EPSRC Doctoral Training Partnership [EP/N509577/1]. leading to a temperature rise and a corresponding pressure rise which propagates as an ultrasound wave [10], [11]. Ultrasound reception can be performed optically using interferometric methods [12]-[15]. One promising method providing high sensitivities and wide bandwidths is the Fabry-Pérot hydrophone [12], [13]. In order to create highly miniaturised imaging devices, both ultrasound generation and reception can be performed at the distal end of optical fibers [16]-[21]. These devices have been shown to provide high resolution imaging and can be used for real-time guidance [17]. Moreover, the use of optics allows the elegant integration of photoacoustic imaging [22]. Here, wavelength-selective composites can be used. These materials absorb light at one wavelength range for ultrasound generation and transmit light at another wavelength range for photoacoustic excitation. This allows for both ultrasound generation and light delivery for photoacoustic excitation to be confined to a single optical fibre, reducing device size and complexity.

Here we demonstrate the use of a low-cost, commercially available, permanent marker ink to fabricate a wavelengthselective composite for dual-modality all-optical ultrasound and photoacoustic imaging. A composite material was fabricated and coated onto the tips of optical fibres to create a miniaturised ultrasound and photoacoustic transmitter. The combination of the transmitter with a plano-concave FabryPérot fiber optic hydrophone was used to demonstrate coregistered imaging using a wire phantom.

\section{METHODS}

A. All-optical ultrasound and photoacoustic transmitter fabrication

A composite solution comprising permanent marker ink (Lumocolor, Staedtler, UK) and polydimethylsiloxane (PDMS) (MED-1000, Polymer Systems Technology, UK) was prepared (Fig. 1(a)). The as bought ink $(1 \mathrm{ml})$ was left exposed in ambient conditions to remove the solvent. Xylene $(1 \mathrm{ml})$ was added to the dry ink to form a solution. Subsequently, the ink solution was manually mixed with PDMS (1 g) to for a homogeneous composite solution. Multimode optical fibers with a $200 \mathrm{~m}$ core diameter (FG200LCC, Thorlabs, UK) were 
a)
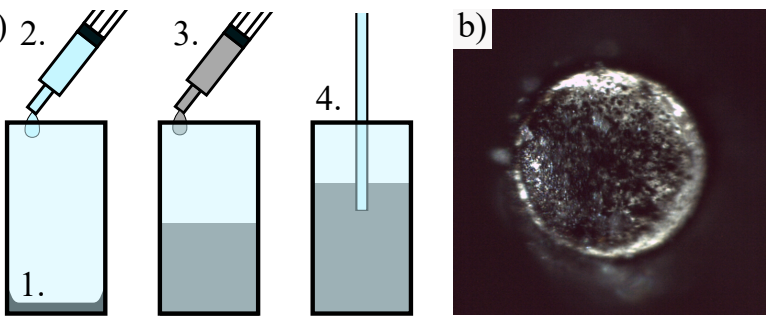

Fig. 1. Fabrication of the ink/PDMS composite. (a) Schematic showing the fabrication process for the ink/PDMS composite coating. 1. Ink left to dry. 2. Xylene added to dry ink. 3. PDMS added to ink solution. 4. Optical fiber dip coated with ink/PDMS composite solution. (b) Ink/PDMS composite fiber optic ultrasound transmitter with through illumination as viewed on a stereomicroscope.

prepared for dipping into the ink solution. The fibers were stripped of their buffer coating at the distal end and cleaved. The cleaved distal end surface was dipped into the composite solution and removed leaving a composite coating on the fiber tip. Subsequently, the coated fibers were stored with the distal end facing up for 24 hours to allow the composite to cure. The fabricated ink/PDMS composite fiber optic ultrasound transmitters were examined using a stereomicroscope (Fig. 1(b)).

\section{B. Ink/PDMS composite characterisation}

The optical absorption within the ink/PDMS composite was characterised. The coated distal end of the fibre was inserted into an integrating sphere (FOIS-1, Ocean Optics, USA) and illuminated through the optical fibre using a broadband white light source (HL-2000-HP-FHSA, Ocean Optics, USA). The output from the integrating sphere was measured using a spectrometer (Maya2000 Pro, Ocean Optics, USA), over a wavelength range of 400 to $1100 \mathrm{~nm}$. The measured optical spectrum was compared to that recorded using a bare optical fiber to find the optical absorption spectrum.

The generated ultrasound characteristics of the ink/PDMS composite fiber optic transmitter were measured. To generate ultrasound a Q-switched pulsed laser (FQ-500-532, Elforlight, $\mathrm{UK}$ ), with a wavelength of $532 \mathrm{~nm}$, a pulse width of $10 \mathrm{~ns}$, and a repetition rate of $100 \mathrm{~Hz}$, was coupled into the optical fiber. The laser fluence was $60 \mathrm{~mJ} / \mathrm{cm}^{2}$ at the coating. The generated ultrasound was measured at $1.5 \mathrm{~mm}$ from the coating using a needle hydrophone (200 $\mu \mathrm{m}$ diameter, Precision Acoustics, UK) with a calibrated range from 1 to $30 \mathrm{MHz}$.

\section{All-optical ultrasound and photoacoustic imaging}

To fabricate an all-optical ultrasound and photoacoustic imaging probe, the ink/PDMS composite fiber optic transmitter was paired with a plano-concave Fabry-Pérot fiber optic hydrophone [12], [13]. Ultrasound was generated using a Qswitched pulsed laser (FQ-500-532, Elforlight, UK), with a wavelength of $532 \mathrm{~nm}$, a pulse width of $10 \mathrm{~ns}$, and a repetition rate of $100 \mathrm{~Hz}$. Photoacoustic excitation was provided by a second Q-switched pulsed laser (SPOT-10-500-1064, Elforlight, UK), with a wavelength of $1064 \mathrm{~nm}$, a pulse width of $2 \mathrm{~ns}$, and a repetition rate of $100 \mathrm{~Hz}$. The Fabry-Pérot
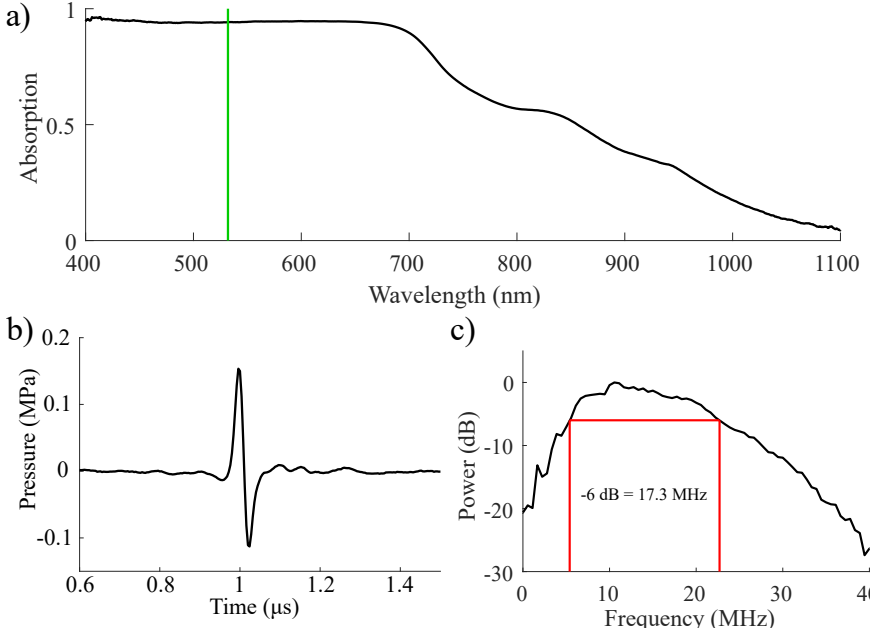

c)

Fig. 2. Optical and ultrasound characteristics of the ink/PDMS composite. (a) Optical absorption spectrum. The vertical green line indicates the wavelength used for ultrasound generation $(532 \mathrm{~nm})$. (b) Ultrasound timeseries measured at $1.5 \mathrm{~mm}$ from the coating. (c) Corresponding ultrasound power spectrum.

ultrasound receiver was interrogated using a continuous wave tuneable laser (Tunics T100S CL, Yenista Optics, France), via a circulator. The reflected optical signal from the FabryPérot cavity was measured using a custom photodetector which split the signal into low- $(<50 \mathrm{kHz})$ and high-frequency $(>500 \mathrm{kHz}$ ) components. The low frequency signal was used to identify the optimum bias wavelength of the FabryPérot cavity. The high-frequency signal was encoded with the received ultrasound signal and was digitized at $100 \mathrm{MS} / \mathrm{s}$ (PCI5142, National Instruments, UK).

Ultrasound imaging and photoacoustic imaging followed the same protocol. Imaging was performed by translating the probe using a motorised stage. The probe was translated in $50 \mu \mathrm{m}$ steps across the imaging region and a signal was recorded at each step. In order to acquire co-registered images, the scan was first performed using the $532 \mathrm{~nm}$ laser for ultrasound generation. Subsequently, the scan was repeated using the $1064 \mathrm{~nm}$ laser for photoacoustic excitation. The received data was processed and reconstructed as described previously [22].

\section{RESUlTS}

\section{A. Optical absorption of ink/PDMS composite}

The optical absorption of the ink/PDMS composite was wavelength-selective (Fig. 2(a)). For wavelengths below $700 \mathrm{~nm}$, the composite exhibited a high optical absorption, with an optical absorption $>90 \%$ at $532 \mathrm{~nm}$. From $700 \mathrm{~nm}$ upwards, the optical absorption decreased and was $<10 \%$ at $1064 \mathrm{~nm}$. This absorption profile allows for absorption of light for ultrasound generation at $532 \mathrm{~nm}$ and transmission of light at longer wavelengths for photoacoustic imaging. 
a)
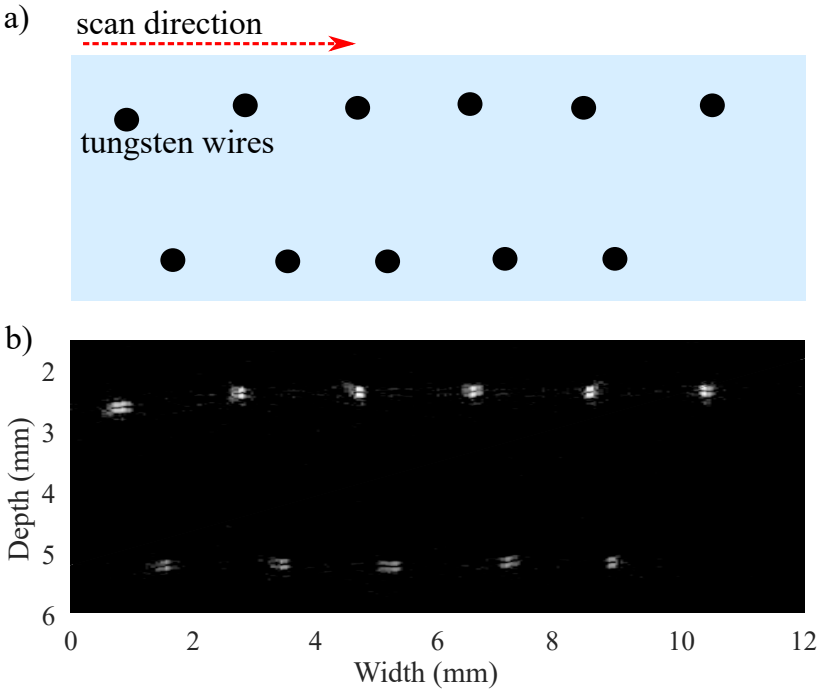

c)
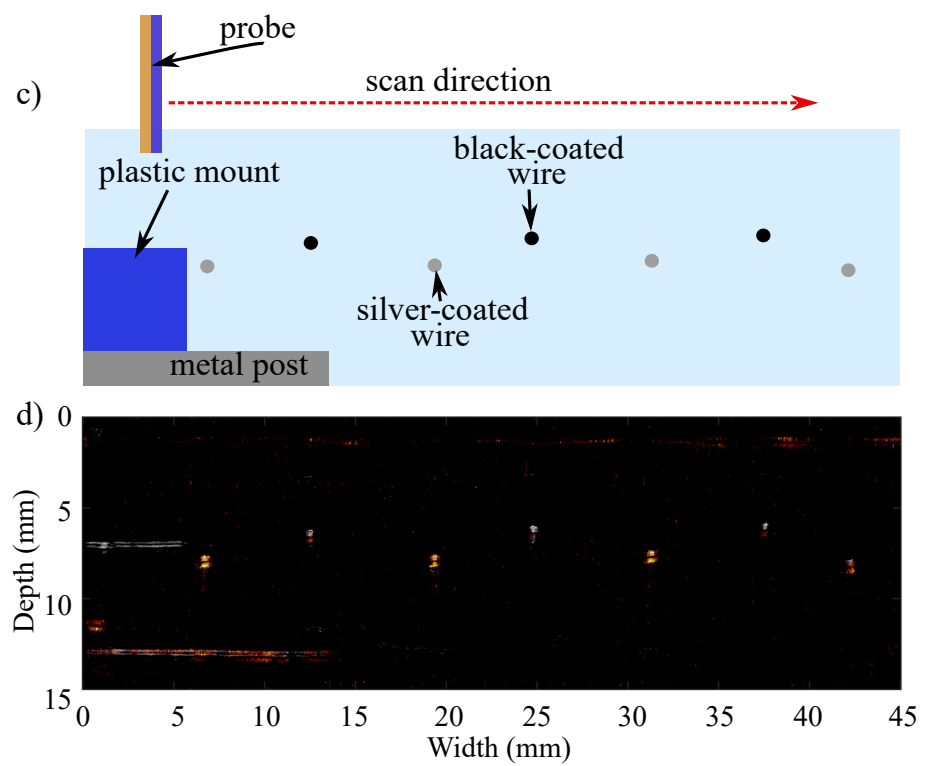

Fig. 3. All-optical ultrasound and photoacoustic imaging of wire phantoms. (a) Schematic of the resolution phantom comprising tungsten wires. (b) All-optical ultrasound image of the tungsten wire resolution phantom. (c) Schematic of the imaging setup and the alternating coated wire phantom. (d) Co-registered all-optical ultrasound (gray) and photoacoustic (hot) image of the alternating coated wire phantom.

\section{B. Ultrasound generation characteristics of the ink/PDMS composite}

The ultrasound generated by the ink/PDMS composite fiber optic transmitters was measured. The generated ultrasound pulse was bipolar in shape and had a peak-to-peak pressure of $266 \mathrm{kPa}$ measured at a distance of $1.5 \mathrm{~mm}$ from the coating surface (Fig. 2(b)). The corresponding power spectrum had a $-6 \mathrm{~dB}$ bandwidth of $17.3 \mathrm{MHz}$ and a central frequency ca. $14 \mathrm{MHz}$ (Fig. 2(c)). These characteristics suggest the fabricated transmitters are suitable for all-optical ultrasound imaging.

\section{All-optical ultrasound and photoacoustic imaging}

To assess the ultrasound imaging resolution, a phantom consisting of tungsten wires ( $27 \mu \mathrm{m}$ diameter) stretched across a plastic mount was imaged (Fig. 3(a,b)). Notably, the tungsten wires produced no photoacoustic signal, thus only the ultrasound image is shown. The resolution was measured as the the full-width at half-maximum of the imaged tungsten wires. The axial resolution was found to be $180 \mu \mathrm{m}$ and the lateral resolution was $100 \mu \mathrm{m}$.

To produce co-registered ultrasound and photoacoustic images a wire phantom consisting of alternating silver- and black-coated copper wires was used. Ultrasound signals were received from all of the wires in the phantom (Fig. 3(c,d)). For the photoacoustic image, the signal from the silver-coated wires was stronger than that of the black-coated wires by $6 \mathrm{~dB}$. The registration between the two images demonstrates the information gained by combining the two modalities.

\section{DISCUSSION AND CONCLUSION}

In this work, a novel dual-modality all-optical ultrasound and photoacoustic transmitter was fabricated. Here, a low- cost wavelength-selective composite was employed to enable ultrasound generation and photoacoustic excitation from a single optical fiber. The composite comprised commercially available materials, namely a permanent marker ink and PDMS. By exciting the coating at one optical wavelength $(532 \mathrm{~nm})$ ultrasound was generated, whilst for wavelengths $>1000 \mathrm{~nm}$, the coating was substantially transparent, allowing photoacoustic excitation light to be transmitted. Transparency in this range is particularly desirable as it is commonly used for photoacoustic imaging [11]. Further, for applications such as intravascular imaging transparency at $1210 \mathrm{~nm}$ is desirable as it coincides with a lipid absorption peak and so can be used to identify lipid in atherosclerotic plaques [22].

The ultrasound generated within the coating on pulsed laser excitation had a peak-to-peak pressure sufficient to enable ultrasound imaging with no averaging. Further, the ultrasound bandwidth led to a high imaging resolution, $180 \mu \mathrm{m}$ in the axial extent and $100 \mu \mathrm{m}$ in the lateral extent. These promising values are sufficient to enable high-fidelity ultrasound imaging. A phantom consisting of alternating silver- and black-coated wires was imaged to demonstrate co-registered dual-modality all-optical ultrasound and photoacoustic imaging with the device. These images demonstrate the advantage of combining modalities in a hybrid device. Ultrasound provided structural contrast, showing the location of all the wires, whilst the photoacoustic image allowed for differentiation between the wire types.

This work demonstrates that low-cost materials can be used to fabricate hybrid, dual-modality imaging devices. Whilst the pressures generated here were sufficient for imaging without the need for averaging, they were low compared with those generated by other PDMS composites [16], [21]. Further work 
is needed to optimize the coating process in order to achieve thinner, homogeneous layers of ink, which integrate well with the PDMS. These optimizations would enable the generation of higher ultrasound pressures and wider bandwidths. Such optimizations may be achieved using different solvents, or coating strategies such as those highlighted previously [22]. It is expected that dual-modality all-optical ultrasound devices will find a wide range of uses in clinical interventions in the near future.

\section{REFERENCES}

[1] Rishi Puri, Matthew I. Worthley, and Stephen J. Nicholls. Intravascular imaging of vulnerable coronary plaque: current and future concepts. Nature Reviews Cardiology, 8(3):131-139, mar 2011.

[2] Christos V. Bourantas, Hector M. Garcia-Garcia, Katerina K. Naka, Antonios Sakellarios, Lambros Athanasiou, Dimitrios I. Fotiadis, Lampros K. Michalis, and Patrick W. Serruys. Hybrid Intravascular Imaging. Journal of the American College of Cardiology, 61(13):1369-1378, apr 2013.

[3] Gijs van Soest, Evelyn Regar, and Antonius F. W. van der Steen. Photonics in cardiovascular medicine. Nature Photonics, 9(10):626-629, oct 2015.

[4] Krista Jansen, Antonius F W van der Steen, Heleen M M van Beusekom, J Wolter Oosterhuis, and Gijs van Soest. Intravascular photoacoustic imaging of human coronary atherosclerosis. Optics Letters, 36(5):597, mar 2011.

[5] Krista Jansen, Gijs van Soest, and Antonius F.W. van der Steen Intravascular Photoacoustic Imaging: A New Tool for Vulnerable Plaque Identification. Ultrasound in Medicine \& Biology, 40(6):1037-1048, jun 2014.

[6] Yingchun Cao, Jie Hui, Ayeeshik Kole, Pu Wang, Qianhuan Yu, Weibiao Chen, Michael Sturek, and Ji-Xin Cheng. High-sensitivity intravascular photoacoustic imaging of lipidladen plaque with a collinear catheter design. Scientific Reports, 6(April):25236, apr 2016.

[7] Shriram Sethuraman, Salavat Aglyamov, James Amirian, Richard Smalling, and Stanislav Emelianov. Intravascular photoacoustic imaging using an IVUS imaging catheter. IEEE Transactions on Ultrasonics, Ferroelectrics and Frequency Control, 54(5):978-986, may 2007.

[8] Bo Wang, Andrei Karpiouk, Doug Yeager, James Amirian, Silvio Litovsky, Richard Smalling, and Stanislav Emelianov. In vivo Intravascular Ultrasound-guided Photoacoustic Imaging of Lipid in Plaques Using an Animal Model of Atherosclerosis. Ultrasound in Medicine \& Biology, 38(12):2098-2103, dec 2012.

[9] Pu Wang, Teng Ma, Mikhail N. Slipchenko, Shanshan Liang, Jie Hui, K. Kirk Shung, Sukesh Roy, Michael Sturek, Qifa Zhou, Zhongping Chen, and Ji-Xin Cheng. High-speed Intravascular Photoacoustic Imaging of Lipid-laden Atherosclerotic Plaque Enabled by a 2-kHz Barium Nitrite Raman Laser. Scientific Reports, 4(1):6889, may 2015.

[15] Shai Ashkenazi, Chung-Yen Chao, L. Jay Guo, and Matthew O'Donnell. Ultrasound detection using polymer microring optical resonator. Applied Physics Letters, 85(22):5418-5420, nov 2004.
[10] B. T. Cox and P. C. Beard. Fast calculation of pulsed photoacoustic fields in fluids using k-space methods. The Journal of the Acoustical Society of America, 117(6):3616-3627, 2005.

[11] Paul Beard. Biomedical photoacoustic imaging. Interface focus, 1(4):602-31, aug 2011.

[12] Edward Z. Zhang and Paul C. Beard. Characteristics of optimized fibre-optic ultrasound receivers for minimally invasive photoacoustic detection. In Alexander A. Oraevsky and Lihong V. Wang, editors, Proc. of SPIE, Photons Plus Ultrasound: Imaging and Sensing, volume 9323, page 932311, mar 2015.

[13] James A. Guggenheim, Jing Li, Thomas J. Allen, Richard J. Colchester, Sacha Noimark, Olumide Ogunlade, Ivan P. Parkin, Ioannis Papakonstantinou, Adrien E. Desjardins, Edward Z. Zhang, and Paul C. Beard. Ultrasensitive plano-concave optical microresonators for ultrasound sensing. Nature Photonics, 11(11):714-719, nov 2017.

[14] Heming Wei and Sridhar Krishnaswamy. Polymer micro-ring resonator integrated with a fiber ring laser for ultrasound detection. Optics Letters, 42(13):2655, jul 2017.

[16] Richard J Colchester, Edward Z Zhang, Charles A Mosse, Paul C Beard, Ioannis Papakonstantinou, and Adrien E Desjardins. Broadband miniature optical ultrasound probe for high resolution vascular tissue imaging. Biomedical Optics Express, 6(4):1502, apr 2015.

[17] Malcolm C. Finlay, Charles A. Mosse, Richard J. Colchester, Sacha Noimark, Edward Z. Zhang, Sebastien Ourselin, Paul C. Beard, Richard J Schilling, Ivan P. Parkin, Ioannis Papakonstantinou, and Adrien E. Desjardins. Through-needle all-optical ultrasound imaging in vivo: a preclinical swine study. Light: Science \& Applications, 6(12):e17103, dec 2017.

[18] E Vannacci, L Belsito, F Mancarella, M Ferri, G P Veronese, A Roncaglia, and E Biagi. Miniaturized fiber-optic ultrasound probes for endoscopic tissue analysis by micro-opto-mechanical technology. Biomedical microdevices, 16(3):415-26, jun 2014.

[19] Erwin J. Alles, Richard J. Colchester, and Adrien E. Desjardins. Adaptive Light Modulation for Improved Resolution and Efficiency in AllOptical Pulse-Echo Ultrasound. IEEE Transactions on Ultrasonics, Ferroelectrics, and Frequency Control, 63(1):83-90, jan 2016.

[20] Xiaotian Zou, Nan Wu, Ye Tian, and Xingwei Wang. Broadband miniature fiber optic ultrasound generator. Optics Express, 22(15):18119, jul 2014.

[21] Sacha Noimark, Richard J Colchester, Ben J Blackburn, Edward Z Zhang, Erwin J Alles, Sebastien Ourselin, Paul C Beard, Ioannis Papakonstantinou, Ivan P Parkin, and Adrien E Desjardins. CarbonNanotube-PDMS Composite Coatings on Optical Fibers for All-Optical Ultrasound Imaging. Advanced Functional Materials, 26(46):8390_ 8396, dec 2016.

[22] Sacha Noimark, Richard J. Colchester, Radhika K. Poduval, Efthymios Maneas, Erwin J. Alles, Tianrui Zhao, Edward Z. Zhang, Michael Ashworth, Elena Tsolaki, Adrian H. Chester, Najma Latif, Sergio Bertazzo, Anna L. David, Sebastien Ourselin, Paul C. Beard, Ivan P. Parkin, Ioannis Papakonstantinou, and Adrien E. Desjardins. Polydimethylsiloxane Composites for Optical Ultrasound Generation and Multimodality Imaging. Advanced Functional Materials, 28(9):1704919, feb 2018. 